\title{
Metrópoli Digital: Una plataforma Web para la inclusión integral de las PyMES, Sociedad y Gobierno en el uso de las Tecnologías de la Información en la región de las Altas Montañas del estado de Veracruz, México
}

Sergio D. Ixmatlahua ${ }^{1}$, Ricardo O. Raygoza ${ }^{1}$, Omar Romero ${ }^{1}$, Fernando Uribe ${ }^{1}$, Efraín J. Vargas $^{2}$

sergio.ixmatlahua@outlook.com,raygoz@gmail.com, isc.romeroo@gmail.com, jonvarg23@ gmail.com

${ }^{1}$ Instituto Tecnológico Superior de Zongolica, Departamento de Sistemas y Computación, Av. Poniente 7, No. 856, Col. Centro, Orizaba, Veracruz, México.

${ }^{2}$ Instituto Tecnológico de Iztapalapa, Av. Telecomunicaciones S/N, Col. Chinamac de Juárez. Iztapalapa, Ciudad de México, México

DOI: 10.17013/risti.e3.43-54

Resumen: Las plataformas de servicios en la nube representan un camino inevitable que tarde o temprano están tomando las ciudades, actualmente se encuentran diversos servicios concentrados en una misma plataforma en diversas ciudades. El desarrollo de sistemas bajo la temática de ciudades virtuales en la Web forma parte de una tendencia que se está implementando. Una plataforma de este tipo ofrece grandes ventajas tanto en la interacción empresas - usuarios - gobierno, como en el efecto detonante que representa el poner a la disposición del público la información detallada de negocios que por sí mismos no cuentan con ningún tipo de servicio que los identifique en Internet. Bajo este escenario es que Metrópoli Digital nace con el objetivo de ofrecer a las PyMES, que no cuentan con recursos ni infraestructura para posicionar su negocio en los medios digitales, una plataforma libre que les permita un posicionamiento digital a través de un modelo de negocio impulsado por las TI.

Palabras clave: Metrópoli Digital, Tecnologías de la Información, Gobernanza de TI, Altas Montañas, comercio electrónico, economía digital.

Digital Metropoli: A Web platform for comprehensive inclusion of SMEs, society and government for using of Information Technology in the Region of the High Mountains of the state of Veracruz, Mexico

Abstract: The services in the cloud represent an inevitable path that cities are implemented. Today, there are several services integrated on the same platform in different cities. The systems development under the theme of virtual cities on the 
Web is part of a trend that is being implemented as a service in the cloud. A platform of this type offers great advantages in interaction enterprises - users - government, to make publicly available detailed business information which themselves do not have any type of service that identifies the Internet. Under this scenario Digital Metropolis is created and its main goal is to proveide the SMEs a free platform that allows them a digital positioning through a business model driven IT, which lack the resources and infrastructure to position your business in digital media.

\section{Introducción}

Con el rápido crecimiento de las redes computacionales, han surgido muchos beneficios para los negocios mediante el uso de las tecnologías Web, es indudable que Internet se ha convertido en un campo con cada vez mayor número de usuarios en todo el mundo, y un lugar idóneo para la publicidad y la promoción de productos y servicios; actualmente muchas transacciones son preferentemente realizadas de manera remota a través del uso de plataformas que funcionan sobre esta gran red de redes. En ese sentido, el uso de la Internet se ha convertido en una necesidad y un derecho humano para garantizar el acceso y el intercambio de información. Sin embargo, aún existen muchos sectores que desconocen estos beneficios y permanecen marginados de estas tecnologías y rezagadas en competitividad con respecto a otros que hacen pleno uso de ellas. Por tanto, en este artículo se describe el trabajo realizado para implementar la plataforma Metrópoli Digital como un esquema de inclusión de las PyMES, Sociedad y Gobierno hacia el uso y aprovechamiento de las Tecnologías de la Información (TI) como parte de la reactivación económica en el estado de Veracruz. Dicha plataforma ofrece diversos servicios y productos a los usuarios tales como localización de negocios, oferta de servicios: clima, trámites y cursos de dependencias gubernamentales, emergencias, asistencia en línea por parte de la Cruz Roja, esto con un modelo de negocio aplicable en cualquier empresa. La plataforma busca catalizar la economía de la región con los servicios digitales proporcionados por la plataforma Metrópoli digital a través de Internet, dando a los negocio principalmente medianos y pequeños un medio digital para aumentar el valor de sus negocios de una forma fácil y accesible.

\section{2. ¿Qué es Metrópoli Digital?}

Con las nuevas estrategias de enseñanza, y con los avances tecnológicos que acompañan el desarrollo social y económico en México, las instituciones educativas de nivel medio superior y superior, han tomado la decisión de apostar por un nuevo modelo educativo en el que los estudiantes lleven una formación académica en participación con las empresas y el sector productivo. Para ello en 2013, la Secretaría de Educación Pública en conjunto con la Secretaría de Gobernación, la Confederación Patronal de la República Mexicana y con la asesoría de la Cámara México - Alemana de Comercio, desarrollaron el Modelo Mexicano de Formación Dual. El cual busca la vinculación armónica entre teoría y práctica, al integrar al estudiante en la empresa para desarrollar competencias profesionales, al mismo tiempo que desarrolla competencias genéricas con el objetivo de obtener una formación integral.

En ese sentido, por un lado, Metrópoli Digital nace como una iniciativa del Instituto Tecnológico Superior de Zongolica, atendiendo al modelo educativo Dual vigente en el 
marco académico de la institución, así como un proyecto integrador en el cual participan estudiantes y docentes de las carreras de Ingeniería en Sistemas Computacionales, Gestión Empresarial y Desarrollo Comunitario de todos los semestres ofertados en la institución. Por otro, Metrópoli Digital es una plataforma Web libre que implementa un modelo de negocio que busca la interacción digital de las PyMES, Sociedad y Gobierno en un esquema integral de gobernanza que permita reactivar el flujo económico a través del uso de las TI como principal medio de intercambio de información y servicios digitales, tales como publicidad, geolocalización de negocios, trámites en línea, sitios personalizados para PyMES, servicios de Gobierno, con la implementación de Aplicaciones Enriquecidas de Internet, RIAs. Ofreciendo una plataforma abierta e intuitiva para mayor accesibilidad de los usuarios no expertos.

Esta plataforma Web busca ser considerada como una Open Web Platform, que de acuerdo a la $\mathrm{W}_{3} \mathrm{C}$ (Hegaret, 2011), una OWP es una plataforma que incluye diversos estándares tales como: HTML5, CSS 2.1, CSS 3, API de geolocalización, eventos DOM, XMLHttpRequest, Web Storage entre otros.

\section{Modelo arquitectónico de software}

Dentro del diseño y desarrollo de sistemas de Información, es de vital importancia tener los cimientos del software de manera que cubra los requisitos no funcionales, los cuales fortalecen las bondades y capacidades de todo sistemas de Información. Para lograr este objetivo, la Ingeniería de Software establece diversos principios para el diseño y desarrollo de Software que abarcan tanto los requisitos funcionales como los no funcionales de un sistema.

The Gang of Four (Jacobson et. at., 2000) mencionan que la arquitectura de un sistema de información define no solo la estructura y su comportamiento, sino también, el uso, funcionalidad, rendimiento, flexibilidad, reutilización, facilidad de comprensión, restricciones y compromisos económicos y tecnológicos, y por supuesto las vistas de las diferentes etapas que conforman el ciclo de vida del software. Otros autores describen a la arquitectura de un sistema software como un conjunto de decisiones de diseño tomadas para un sistema (Tahuiton, 2011).

En la definición de una arquitectura existen diferentes patrones arquitectónicos que facilitan la estructuración de los componentes de software que serán parte de un sistema. Entre los patrones arquitectónicos más conocidos, están los patrones: Modelo - Vista - Controlador, Modelo - Vista - Vista - Modelo, Cliente - Servidor; los cuales son los mayormente utilizados en desarrollos de aplicaciones para ambientes Web. Sin embargo, se ajustan de acuerdo a las necesidades de la arquitectura, pudiendo formar una arquitectura híbrida, en donde más de un patrón sea utilizado.

De acuerdo a lo anterior, y al intenso trabajo de los Stakeholders en el proyecto de Metrópoli Digital, se ha propuesto una arquitectura que integra un patrón híbrido en capas, conformado por los patrones; M-V-C, Cliente - Servidor, ya que son los patrones más importantes que soportan la estructura principal de Metrópoli, el cual incluye el uso de Apps externas para el consumo de servicios a través de la arquitectura RESTfull. 
En la figura 1, se presenta el diseño arquitectónico propuesto para la plataforma, que de acuerdo a la técnica de Ingeniería de Software 4 + 1 vistas (Jacobson et. at., 2000), en su vista de desarrollo, se contempla los siguientes componentes:

Capas de soporte:

$>$ DataBase MySQL

$>$ Web Server

$\bigcirc$ Model Layer

- BusinessModelModel

- ODBConnection

- RecommendationEngine

$\bigcirc$ Controller Layer

- JSONRequestController

- XMLRequestController

- WebServicesRepository

- BusinessModelControllers

$>$ Client

$\bigcirc$ View Layer

- WebMetropoliApp

- MovilNativeApp

- CrossPlatformApp

> ExternalServices

$\bigcirc$ API Geolocation

$\bigcirc$ API WeatherChannel

○ APIjQuery

$\bigcirc$ WSGovernment

A continuación se describe cada uno de los componentes que integran la arquitectura de Metrópoli Digital. 


\section{Model Layer}

Esta capa está encargada de la persistencia de la información, almacenamiento y suministro hacia la capa de control. Permite una comunicación directa con el SGBD, realizando operaciones de acuerdo a las decisiones de la capa de control. El BusinessModel es una representación abstracta de una organización o negocio en otras palabras, describe cómo funciona el negocio, o más específicamente cómo se realizan las actividades o tareas (Thomas, Martin, 2003).

BusinessModel. Representa el núcleo de la arquitectura, con funciones tales como operaciones, toma de decisiones y gestión de peticiones, cubriendo las características más representativas que conforman el objetivo de la plataforma. Este modelo busca representar la función de los procesos administrativos y operacionales que están sustentados en la sección 4 bajo el modelo CANVAS.

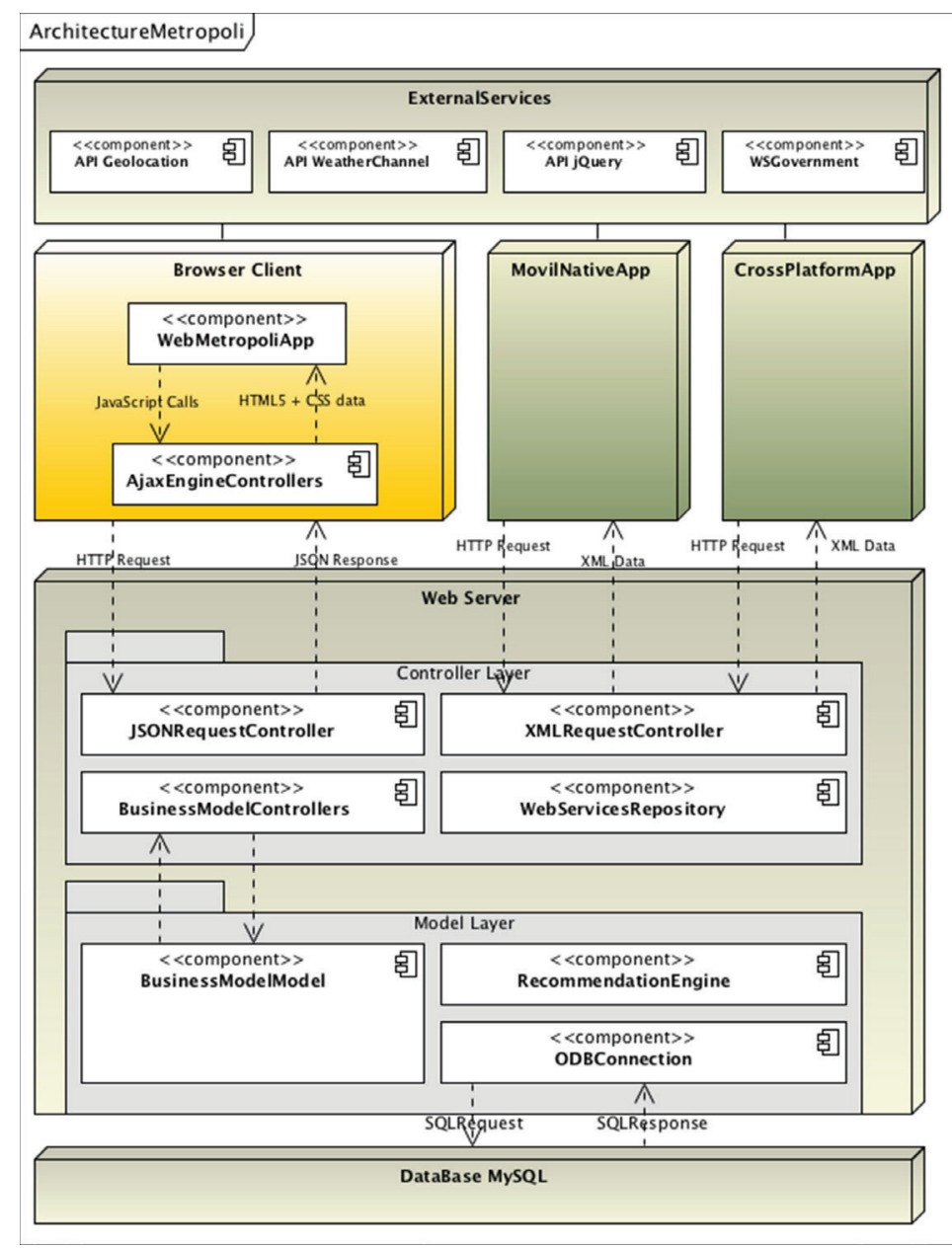

Figura 1 - Arquitectura de Metrópoli Digital 
El ODBConnection, es uno de los componentes medulares para la gestión de la persistencia de la información. Este componente permite la interacción del núcleo de la plataforma con el SGBD, es decir, contiene todas las funciones que permiten acceder a los datos, tales como la abstracción de la base de datos y el acceso a datos. En la Figura 1, se observa la comunicación del ODBConnection con el BusinessModelModel, así como hacia el SGBD.

\section{Controller Layer}

Esta es la capa de control que está encargada de procesar las interacciones del usuario y realizar los cambios apropiados en el modelo y vista. Dentro de las tarea principales de esta capa, está la gestión de las peticiones del usuario y manipulación de modelo. El Controller Layer está dividido en 4 subcontroles: JSONRequestController, XMLRequestController, WebServicesRepository, BusinessModeControllers.

JSONRequestController. Se encarga de manipular las peticiones del usuario a través del uso de HttpRequest, dichas peticiones son enviadas por el cliente (HTML y Ajax), validadas y redirigidas hacia la capa de modelo, y una vez procesadas, crear la estructura JSON correspondiente para generar la respuesta a la petición de las vistas.

XMLRequestController. Tiene la responsabilidad de recibir las peticiones que serán generadas desde las app móviles. El componente recibe las peticiones basadas en XML, tales como SOAP (W3C, 200) que soporta WSDL y REST utilizando JSON ó XML (Fielding, 2000).

BusinessModelControllers. Este componente da soporte completo al BusinessModeloModel en la capa de modelo, debido a que realiza las validaciones, toma de decisiones sobre que acciones deben ser llamadas desde el modelo. El componente contiene clases escrita en PHP del lado del servidor, las cuales trabajan en conjunto con el JSONRequest.

WebServicesRepository. Contendrá un compendio de Web Services basados en SOAP y REST, con el objetivo de proveer acceso a las App móviles que harán uso de los datos de Metrópoli Digital. En ese sentido, el componente tiene la labor de asegurar la interconectividad con el componente XMLRequestController, el cual se encargará de seleccionar el Web Services indicado de acuerdo con la petición.

\section{View Layer}

La capa ViewLayer es la parte del sistema de más alto nivel y la que se encarga de presentar la interfaz de usuario, dicha capa está implementada haciendo uso Aplicaciones Enriquecidas de Internet RIA (por su siglas en inglés Rich Internet Applications) que le dan una experiencia mucho más intuitiva y accesible a los usuarios. Esta capa contempla 3 diferentes clientes, los cuales se mencionan a continuación.

WebMetropoliApp. Este componente está disponible vía Internet, para poder accederse a través de un navegador Web, dicho complemento es una vista que cuenta con la parte visible de la plataforma, está construida haciendo uso de frameworks RIA's tales como jQuery, HTML5, CSS 3, entre otros. En una primera implementación la plataforma se 
desarrolló usando el lenguaje PHP. Sin embargo, se busca la mejora continua, por lo que se buscará migrar la plataforma a un lenguaje más robusto como Java.

MovilNativeApp. Este elemento, es un componente que representa a una aplicación móvil nativa, la cual estará hecha en Android, por lo que permitirá obtener información de los servicios que Metrópoli Digital ofrece, desde un dispositivo móvil. La aplicación accederá a los datos a través del consumo de Web Services.

CrossPlatformApp. Este componente representa a un cliente basado en tecnologías multi-teléfono, con el objetivo de poder abarcar plataformas tales como IOS, Blackberry, Windows Phone, WebOS, entre muchos otros. Para ellos, se desarrollará una aplicación con el uso del framework PhoneGap que está basado en HTML5, CSS 3, jQuery, el cual cubre dicho requisito y hace un desarrollo fácil y rápido.

\section{Modelo de negocios de Metrópoli Digital}

El modelo de negocio que soporta la estructura empresarial de Metrópoli Digital, está basada en la propuesta formulada por Alexander Osterwalder en el año 2004, y la cual es una herramienta para colocar de manera estratégica la planeación de un modelo de negocios, ya que Metrópoli Digital busca la correcta gobernanza de los elementos, para desarrollar una sinergia y poder lograr así los objetivos deseados en el proyecto. En figura 2 nos muestra de manera gráfica la mitad del modelo.

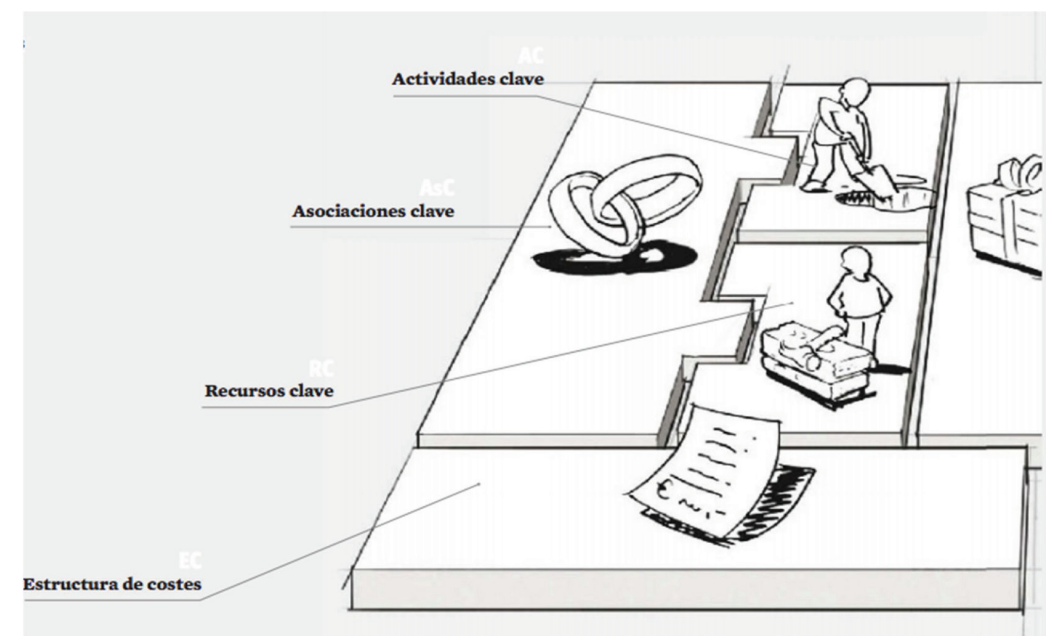

Figura 2 - Primera fase del modelo CANVAS

Segmentos de Mercado Según (Osterwalder, 2011) Los clientes son el centro de cualquier modelo de negocio, ya que ninguna empresa puede sobrevivir durante mucho tiempo si no tiene clientes (rentables), y es posible aumentar la satisfacción de los mismos agrupándolos en varios segmentos con necesidades, comportamientos y atributos comunes. Un modelo de negocio puede definir uno o varios segmentos de mercado, 
ya sean grandes o pequeños. Las empresas deben seleccionar, con una decisión fundamentada, los segmentos a los que se van a dirigir y, al mismo tiempo, los que no tendrán en cuenta. Una vez que se ha tomado esta decisión, ya se puede diseñar un modelo de negocio basado en un conocimiento exhaustivo de las necesidades específicas del cliente objetivo. En la figura se muestra el complemento del modelo.

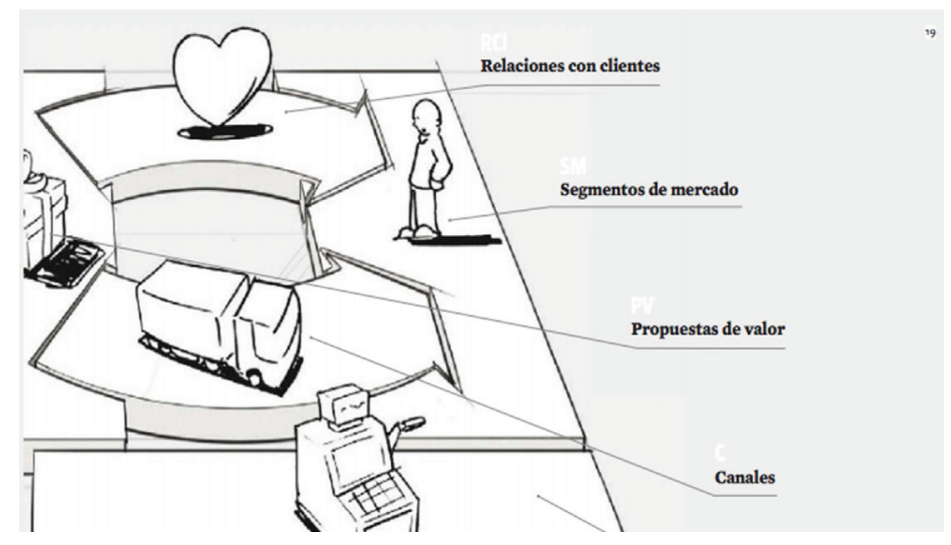

Figura 3 - Complementos del modelo de negocios

Propuesta de valor. Según (Osterwalder, 2011) En este módulo se describe el conjunto de productos y servicios que crean valor para un segmento de mercado específico. La propuesta de valor es el factor que hace que un cliente se decante por una u otra empresa; su finalidad es solucionar un problema o satisfacer una necesidad del cliente. Las propuestas de valor son un conjunto de productos o servicios que satisfacen los requisitos de un segmento de mercado determinado. En este sentido, la propuesta de valor constituye una serie de ventajas que una empresa ofrece a los clientes. Algunas propuestas de valor pueden ser innovadoras y presentar una oferta nueva o rompedora, mientras que otras pueden ser parecidas a ofertas ya existentes e incluir alguna característica o atributo adicional.

Canales según (Pigneur, 2011), Los canales de comunicación, distribución y venta establecen el contacto entre la empresa y los clientes. Son puntos de contacto con el cliente que desempeñan un papel primordial en su experiencia.

Los canales tienen, entre otras, las funciones siguientes:

- Dar a conocer a los clientes los productos y servicios de una empresa

- Ayudar a los clientes a evaluar la propuesta de valor de una empresa

- Permitir que los clientes compren productos y servicios específicos

- Proporcionar a los clientes una propuesta de valor

- Ofrecer a los clientes un servicio de atención posventa

Los canales de Distribución. De acuerdo a (Kotler, 2008) son los elementos del negocio por el cual pasan los productos y/servicios desde su fabricación, hasta su entrega 
como un servicios o productos funcional. Los canales de distribución que se utilizan en Metrópoli Digital, se muestran en la figura 4, de esta manera se puede verificar cuales son los elementos que forma una cadena de distribución de los servicios y/o productos.

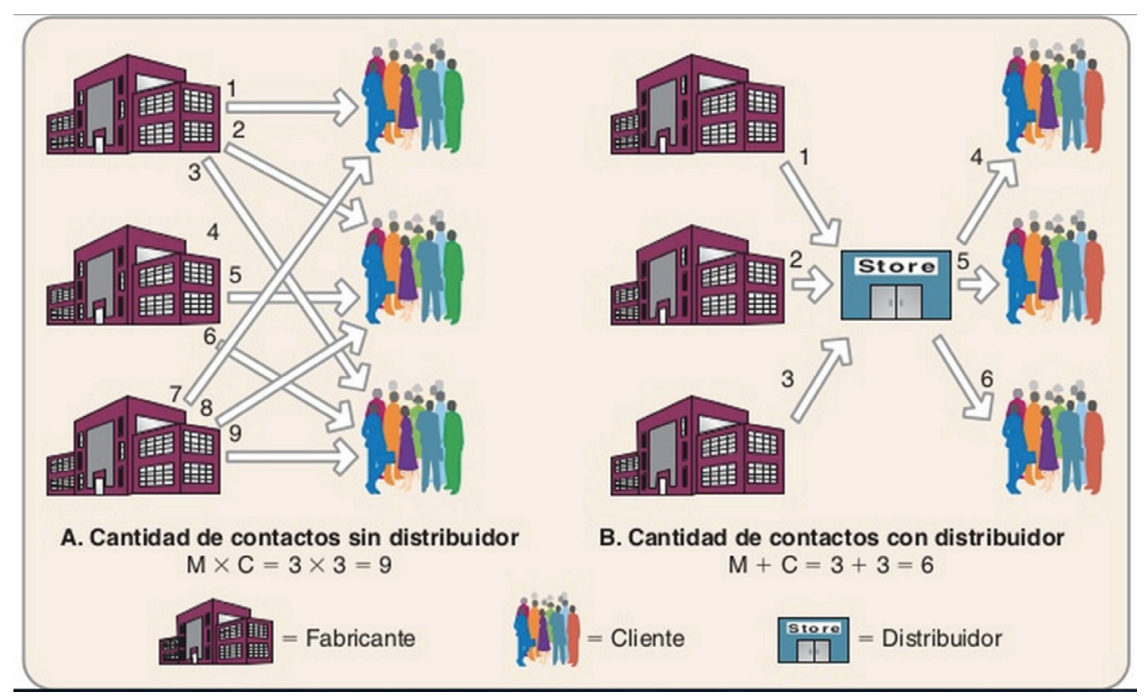

Figura 4 - Canales de distribución para Metrópoli Digital

Relaciones con los Clientes, según (Pigneur, 2011) En este módulo se describen los diferentes tipos de relaciones que establece una empresa con determinados segmentos de mercado. Las empresas deben definir el tipo de relación que desean establecer con cada segmento de mercado. La relación puede ser personal o automatizada. Las relaciones con los clientes pueden estar basadas en los fundamentos siguientes: - Captación de clientes. • Fidelización de clientes. • Estimulación de las ventas (venta sugestiva).

Fuentes de Ingresos, según (Pigneur, 2011) el presente módulo se refiere al flujo de caja que genera una empresa en los diferentes segmentos de mercado (para calcular los beneficios, es necesario restar los gastos a los ingresos).

Un modelo de negocio puede implicar dos tipos diferentes de fuentes de ingresos: 1. Ingresos por transacciones derivados de pagos puntuales de clientes. 2. Ingresos recurrentes derivados de pagos periódicos realizados a

Si los clientes constituyen el centro de un modelo de negocio, las fuentes de ingresos son sus arterias. Las empresas deben preguntarse lo siguiente: ¿̇por qué valor está dispuesto a pagar cada segmento de mercado? Si responde correctamente a esta pregunta, la empresa podrá crear una o varias fuentes de ingresos en cada segmento de mercado. Cada fuente de ingresos puede tener un mecanismo de fijación de precios diferente: lista de precios fijos, negociaciones, subastas, según mercado, según volumen o gestión de la rentabilidad. Cambio del suministro de una propuesta de valor o del servicio postventa de atención al cliente. 
Recursos Clave. En este módulo se describen los activos más importantes para que un modelo de negocio funcione. Todos los modelos de negocio requieren recursos clave que permiten a las empresas crear y ofrecer una propuesta de valor, llegar a los mercados, establecer relaciones con segmentos de mercado y percibir ingresos. Cada modelo de negocio requiere recursos clave diferentes. Un fabricante de microchips necesita instalaciones de producción con un capital elevado, mientras que un diseñador de microchips depende más de los recursos humanos.

Los recursos clave pueden ser físicos, económicos, intelectuales o humanos. Además, la empresa puede tenerlos en propiedad, alquilarlos u obtenerlos de sus socios clave.

Actividades Clave, según (Pigneur, 2011) En el presente módulo se describen las acciones más importantes que debe emprender una empresa para que su modelo de negocio funcione. Todos los modelos de negocio requieren una serie de actividades clave. Estas actividades son las acciones más importantes que debe emprender una empresa para tener éxito, y al igual que los recursos clave, son necesarias para crear y ofrecer una propuesta de valor, llegar a los mercados, establecer relaciones con clientes y percibir ingresos. Además, las actividades también varían en función del modelo de negocio.

Asociaciones Clave, según(Pigneur,2011) En estemódulo se describela red deproveedores y socios que contribuyen al funcionamiento de un modelo de negocio. Las empresas se asocian por múltiples motivos y estas asociaciones son cada vez más importantes para muchos modelos de negocio. Las empresas crean alianzas para optimizar sus modelos de negocio, reducir riesgos o adquirir recursos. Podemos hablar de cuatro tipos de asociaciones:

1. Alianzas estratégicas entre empresas no competidoras.

2. Coopetición: asociaciones estratégicas entre empresas competidoras.

3. Joint ventures: (empresas conjuntas) para crear nuevos negocios.

4. Relaciones cliente-proveedor para garantizar la fiabilidad de los suministros.

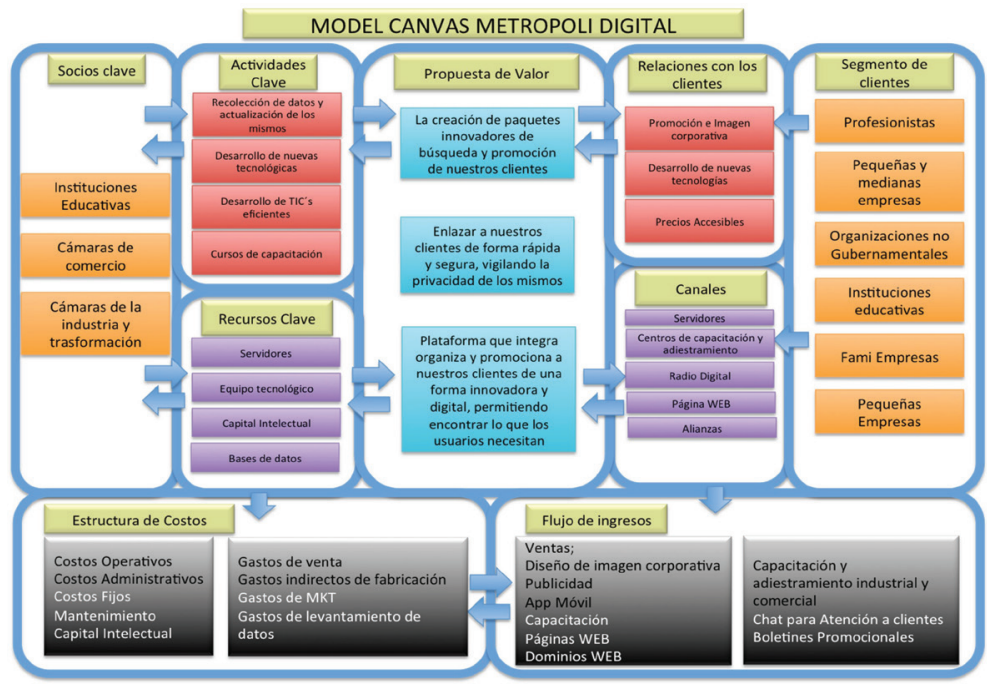

Figura 5 - Modelo CANVAS Metrópoli Digital 
Según (Porter, 2006) dice que el punto inicial para el análisis de costos consiste en definir una cadena de valor y en asignarles a las actividades los costos operativos y los activos. Las actividades de la cadena contiene ambas cosas en forma de capital fijo y de trabajo. Los insumos comprados forman parte del costo de cada actividad, pudiendo afectar a los costos operativos (insumos operativos comprados) y a los activos (activos adquiridos). La necesidad de asignar activos a las actividades de valor se debe a que el total de activos en una actividad y la eficiencia de su utilización a menudo son importantes para el costo de ellas.

Nuestro modelo CANVAS se ve reflejado las actividades de valor como las claves identificando los recursos clave en la figura 5 .

\section{Trabajo Actual}

Metrópoli Digital, al ser un proyecto integrador desarrollado por un instituto educativo y trabajado por estudiantes y docentes de manera colaborativa, esta dividido en tres etapas.

En la Tabla 1, se aprecia cada una de las fases planificadas a desarrollar, cada una de ellas contempla aproximadamente un 33\% del total del proyecto. En ese sentido, Metrópoli Digital está comenzando con la segunda etapa, puesto que se han terminado cada una de las actividades de la primera fase, las cuales eran vitales para la segunda etapa.

Tabla 1 - Fases de implementación de Metrópoli Digital

\begin{tabular}{|c|c|c|}
\hline Logística y estratificación & Optimización de servicios & Expansión \\
\hline $\begin{array}{l}\text { - Análisis, diseño y desarrollo } \\
\text { de la plataforma Web de } \\
\text { Metrópoli Digital. }\end{array}$ & $\begin{array}{l}\text { - Implementación de un sistema de } \\
\text { recomendación de negocios }\end{array}$ & $\begin{array}{l}\text { - Implementación de } \\
\text { Metrópoli Digital en la Zona } \\
\text { Metropolitana Poza Rica. }\end{array}$ \\
\hline $\begin{array}{l}\text { - Logistica y estratificación de } \\
\text { los municipios de la región } \\
\text { de las Altas Montañas. }\end{array}$ & $\begin{array}{l}\text { Desarrollo de una app móvil } \\
\text { multiplataforma como medio de } \\
\text { expansión }\end{array}$ & $\begin{array}{l}\text { - Implementación de } \\
\text { Metrópoli Digital en la zona } \\
\text { Metropolitana Veracruz. }\end{array}$ \\
\hline $\begin{array}{l}\text { - Captura de datos } \\
\text { georeferenciados de los } \\
\text { negocios. }\end{array}$ & $\begin{array}{l}\text { Desarrollo de un repositorio } \\
\text { de Web Services para ofrecer } \\
\text { interconecctividad a otras } \\
\text { plataformas de comunicación }\end{array}$ & $\begin{array}{l}\text { - Implementación de } \\
\text { Metrópoli Digital en la zona } \\
\text { Metropolitana Cordoba. }\end{array}$ \\
\hline $\begin{array}{l}\text { - Publicación de los } \\
\text { contenidos en la plataforma }\end{array}$ & $\begin{array}{l}\text { Digitalización de los trámites y } \\
\text { servicios de los municipios del la } \\
\text { región de las Altas Montañas del } \\
\text { estado de Veracruz }\end{array}$ & \\
\hline $\begin{array}{l}\text { - Revisión e integración de } \\
\text { los servicios digitales en } \\
\text { externos. }\end{array}$ & & \\
\hline
\end{tabular}

En esta segunda fase se pretende desarrollar e implementar un sistema de recomendación de negocios basados en las visitas y comportamiento de los usuarios y ranquin de negocios, así como el desarrollo de una App móvil que permita a los tener un medio de localización de servicios accesibles desde cualquier dispositivo móvil. Se pretende la implementación de un repositorio de Web Services para proveer de servicios a terceros (clima, transito y vialidad, salud y emergencias, turismo, cultura). 
En la tercera y última fase, se buscarán integrar a otras zonas metropolitanas del estado de Veracruz, tales como la zona Metropolitana Xalapa, Córdoba y Poza Rica.

Por tanto, Metrópoli Digital busca ser una plataforma a nivel estatal, que acerque a las PyMES al uso de las TI con el objetivo de posicionar los negocios a través de Internet.

\section{Referencias}

Jacobson, I., Booch, G., Rumbaugh, J. (2000). El proceso unificado de desarrollo de software. Rational Software Corporation. Pearson Addison Wesley. Madrid.

Osterwalder, A. (2011). Business Model Generation. (S. L. Centro Libros PAPF, Ed.) Barcelona, España: Grupo Planeta.

Porter, Michael E. 1991. La ventaja competitiva de las naciones. Buenos Aires, Argentina: Vergara. - (2006). Ventaja competitiva. Quinta reimpresión. México: CECSA. (2009). Ser competitivo. Barcelona, España: Ediciones DEUSTO.

Tahuiton Mora, J. (2011). Arquitectura de software para aplicaciones Web. Centro de Investigación y de Estudios Avanzados del Instituto Politécnico Nacional. México, D.F.

Fielding, R. T. (2000). Architectural Styles and the Design of Network-based Software Architectures. Cap. 5. Universidad de California, Irvine, USA.

W3C Working Group. (2000). Simple Object Access Protocol. 2014/07/25, de World Wide Web Consortium Sitio web: http://www.w3.org/TR/2000/NOTESOAP-20000508/

Philippele Hegaret. (2011). "10o Specifications for the Open Web Platform and Counting”. 01-29-2015, W3C. http://www.w3.org/QA/2011/01/100_specifications_for_the_ ope.html

Javier Eguiluz. (2008). Introducción a AJAX. España: LibrosWeb.es http://librosweb. es/libro/ajax/.

Douglas K Barry. (2015). Web Services Explained. 16-03-2015, de Barry \& Associates, Inc. Sitio web: http://www.service-architecture.com/articles/web-services/web_ services_explained.html.

SEGOB. (2015). "SEP y Coparmex firman convenio de colaboración para impulsar el Modelo Mexicano de Educación Dual en bachillerato". 24/03/2015. Secretaría de Gobernación. Sitio Web: http://www.conaliteg.gob.mx/index. php?view $=$ article\&id $=255$.

Thomas, D. Martin, J. (2003). Process Modeling for E-Business. 20/o3/2015, de George Mason University. 